\title{
Prescribing community psychiatric nurses
}

\author{
Chris Fear, Registrar in Psychiatry, North Wales Hospital, Denbigh, Clwyd LL16 5SS; \\ and GReg Wilkinson, Professor of Psychiatry, The London Hospital Medical \\ College, London E1 2AD
}

Community psychiatric nurses (CPNs) fulfil an important role in caring for people with mental disorders in the community. They provide a monitoring and information service for patients and relatives and form a link between general practitioners (GPs) and psychiatrists. Some CPNs provide advice to GPs about prescribing psychotropic drugs. We studied the extent of this activity in a geographically-defined district and the views of GPs, CPNs and psychiatrists towards it.

\section{The study}

Ten randomly selected GPs, all ten CPNs, and four senior psychiatrists from north Clwyd were interviewed using a semi-structured questionnaire concerning CPN prescribing of psychotropic drugs. CPNs were questioned about training in clinical psychopharmacology and knowledge of psychotropic drugs.

\section{Findings}

All GPs and CPNs reported at least monthly contact, and six GPs reported having weekly contact with CPNs.

\section{Advice about psychotropic drugs}

Only one GP reported seeking advice from a CPN (regarding adverse effects of a particular drug). By contrast, eight CPNs reported that they had been asked by GPs for advice concerning drug management: five said that they were asked by GPs for advice about psychotropic drugs at least weekly, and three others were asked for advice less often.

The advice sought by GPs from CPNs mainly concerned antidepressants and oral and depot neuroleptics, and included indications for use, dose and duration of treatment, adverse effects and appropriate alternative preparations available.

Nine CPNs thought that it was appropriate for GPs to seek their advice about psychotropic medication because of their detailed knowledge of patients' responses.

\section{Advice on psychotropic medication sought by patients and relatives}

Seven GPs reported that they never referred patients or relatives to CPNs for advice on psychotropic medication; three said that they rarely did so.

All CPNs reported frequent, often daily, requests for advice from patients and their relatives on all aspects of clinical management, chiefly about adverse effects and likely duration of drug treatment.

\section{CPNs, psychopharmacology and psychotropic drugs}

One CPN had received post-basic instruction in clinical psychopharmacology. Others reported obtaining their knowledge from the British National Formulary, pharmaceutical representatives, the hospital pharmacy, and nursing journals.

Seven CPNs thought that they were qualified to advise GPs, patients and relatives about psychotropic medication. Four believed that this activity was appropriate if restricted to nursing implications for the patient. The other three CPNs considered themselves unqualified to give advice to GPs regarding psychotropic drugs, though two were happy to advise patients and relatives.

\section{Future training for CPNs}

Eight GPs indicated that CPNs should receive training in clinical psychopharmacology but qualified the extent to which CPNs should be able to give advice about psychotropic drugs. Four thought that CPNs should only advise on a limited range of preparations; two thought that CPNs should be able to advise on any psychotropic preparation; one stated that CPNs should provide advice only to patients and relatives; and one thought that providing advice on medication was not part of the nursing role. Two GPs were satisfied with the present situation.

All CPNs thought that they required training in clinical psychopharmacology: six thought that they should only provide advice on a limited range of psychotropic drugs and four thought that no such restriction was necessary.

Psychiatrists held various opinions: one was satisfied with the present situation and one thought that 
CPNs were not qualified to give advice concerning drug management. The other two gave qualified support for advice to be given by CPNs for a limited range of psychotropic preparations, or for all psychotropic drugs after instruction and training in clinical psychopharmacology. All agreed that any advice given by CPNs should be to patients and their relatives and not to GPs.

\section{CPN prescribing of psychotropic drugs}

Seven GPs supported the prescribing of psychotropic medication by CPNs: four suggested that this could take place under a GP's or a consultant psychiatrist's supervision and three thought that prescribing by CPNs should be under GP supervision only. Three GPs disagreed with the prescribing of psychotropic medication by CPNs.

Eight CPNs supported their prescribing of psychotropic medication: two advocated prescribing under supervision from a GP or a consultant psychiatrist; four thought that such prescribing should be under GP supervision only; one thought that prescribing should either be unsupervised or supervised by a consultant psychiatrist; another upheld unsupervised prescribing. All believed that only a limited range of psychotropic drugs should be prescribed by CPNs.

Two psychiatrists disagreed with CPN prescribing on the grounds that they lacked knowledge of general medicine and therapeutics. The other two saw a limited role for CPNs in altering doses and timing of existing medication rather than initiating drug therapy. They thought that GP supervision would probably be the most satisfactory, with consultants providing back-up. Both thought that $\mathrm{CPN}$ involvement in prescribing should be limited to short-term use of hypnotics and anxiolytics.

\section{Comment}

Doctors' time could be saved if CPNs with appropriate post-basic training were permitted to prescribe (or to modify the timing and dose prescribed) from a limited list of psychotropic drugs.

The Report of the Advisory Group on Nurse Prescribing (1989) accepted the basic concept of nurse prescribing. They concluded that it would not be appropriate for CPNs to become initial prescribers' due to their dual responsibility to hospitalbased and primary health teams, the consequent problems of co-ordination and communication, and the possibility of their coming under unacceptable pressure from some of the clients with whom they had formed a close relationship.

The Advisory Group recommended that CPNs should be afforded the responsibility of prescribing in the case of patients within a patient-specific clinical protocol requiring the modification of timing and dose of medication. The majority of comments from CPNs questioned in our study showed irritation at having to contact a doctor prior to making simple changes in the timing of psychotropic medication.

In the USA, doctors' assistants, nurse practitioners, and optometrists have, over the last 20 years, all gained limited drug prescribing privileges. In August, 1990 the American Department of Defense began a training programme, initially for two psychologists, to enable participants to prescribe four drugs used in psychiatry: diazepam, haloperidol, benztropine mesylate and chlorpromazine (Rhein, 1990). If psychologists with appropriate training may prescribe psychotropic drugs, why not also CPNs, who already seem to be having an extensive, direct influence on the psychotropic drug management of people with mental disorder?

\section{References}

Report of the Advisory Group on Nurse Prescribing (1989) London: Department of Health.

Rhein, R. (1990) Prescribing psychologists. British Medical Journal, 301, 356. 